\title{
The HVEM network: new directions in targeting novel costimulatory/co-inhibitory molecules for cancer therapy Christine Pasero ${ }^{1,2,3}$, Daniel E Speiser ${ }^{4}$, Laurent Derré ${ }^{5}$ and Daniel Olive ${ }^{1,2,3}$
}

\begin{abstract}
The regulation of the immune system is controlled by many cell surface receptors. A prominent representative is the 'molecular switch' HVEM (herpes virus entry mediator) that can activate either proinflammatory or inhibitory signaling pathways. HVEM ligands belong to two distinct families: the TNF-related cytokines LIGHT and lymphotoxin- $\alpha$, and the Ig-related membrane proteins BTLA and CD160. HVEM and its ligands have been involved in the pathogenesis of various autoimmune and inflammatory diseases, but recent reports indicate that this network may also be involved in tumor progression and resistance to immune response. Here we summarize the recent advances made regarding the knowledge on HVEM and its ligands in cancer cells, and their potential roles in tumor progression and escape to immune responses. Blockade or enhancement of these pathways may help improving cancer therapy.
\end{abstract}

\footnotetext{
Addresses

${ }^{1}$ Aix-Marseille Université, F-13284, Marseille, France

${ }^{2}$ Institut National de la Santé et de la Recherche Médicale, UMR 1068 , Centre de Recherche en Cancérologie de Marseille, F-13009, Marseille, France

${ }^{3}$ Institut Paoli Calmettes, IBiSA Cancer Immunomonitoring Platform, F13009, Marseille, France

${ }^{4}$ Clinical Tumor Immune-Biology Unit, Ludwig Institute for Cancer Research of the University of Lausanne, Switzerland

${ }^{5}$ Urology Research Unit, Department of Urology, Lausanne University Hospital (CHUV), Switzerland
}

Corresponding author: Olive, Daniel (Daniel.olive@marseille.inserm.fr, Daniel.Olive@inserm.fr)

Current Opinion in Pharmacology 2012, 12:478-485

This review comes from a themed issue on Immunomodulation

Edited by Stephen G Ward and Luke AJ O'Neill

For a complete overview see the Issue and the Editorial

Available online 23th March 2012

1471-4892/\$ - see front matter, (C) 2012 Elsevier Ltd. All rights reserved.

http://dx.doi.org/10.1016/j.coph.2012.03.001

\section{Introduction}

Co-signaling molecules include positive and negative receptors that allow regulation and fine tuning of the immune response. They consist of two superfamilies, classified based on their structure: the tumor necrosis factor receptor (TNFR) family characterized by cystein-rich domains (CRDs) in the extracellular portion, and the immunoglobulin (Ig) superfamily, or 'CD28 and B7 family', whose members contain Ig-variable-like extracellular domains. The TNFR superfamily comprises important players in costimulation and coinhibition, including 4-1BB, OX40, CD27, CD40 and HVEM. Concerning the Ig superfamily, apart from CD28 and ICOS that deliver co-stimulatory signals to T cells, other members of this family are involved in inhibiting or attenuating TCR-mediated activation. These co-inhibitory Ig members include CTLA-4, BTLA, PD1, and the recently discovered molecule CD160.

Here we focus on members of these families that attracted much attention during these past few years: the TNF receptor HVEM (herpes virus entry mediator), a molecular switch between proinflammatory and inhibitory signaling and the HVEM ligands, which are LIGHT (TNFSF14, lymphotoxin-like, exhibits inducible expression, and competes with herpes simplex virus (HSV) glycoprotein D for $\underline{H}$ EM, a receptor expressed by $\underline{T}$ lymphocytes), BTLA (B and T lymphocyte attenuator) and CD160. Given the importance of HVEM and its ligands in the physiopathology of immune regulation, it is now clear that the dysregulation of this network contributes to various diseases. Many reviews have highlighted the interest of manipulating these pathways in the context of autoimmunity and transplantation $\left[1,2^{\bullet}, 3^{\bullet}\right]$. Recent studies of outstanding interest have also described abnormal expression of these co-stimulatory molecules in tumor cells $\left[4^{\bullet}, 5^{\bullet}, 6^{\bullet \bullet}\right]$. Moreover, clinical trials targeting members of these families, the co-inhibitory $\mathrm{Ig}$ molecules CTLA-4 and PD-1 (programmed death-1), have already given promising results in patients with melanoma, renal cell and prostate carcinoma, and non-Hodgkin's lymphoma [7]. In the same way, there is clear interest in evaluating the potential role of HVEM and its ligands in cancer therapy.

In this review we focus on (i) the expression patterns and function of HVEM and its ligands on normal tissues, (ii) the expression of these molecules in tumor cells and their involvement in tumor development and resistance to cancer, (iii) and the possible exploitation of these pathways for novel therapies using antibodies or recombinant proteins.

\section{Around the HVEM network TNF-related and unconventional ligands}

HVEM, first discovered as the entry route for HSV has the particularity to connect the two superfamilies. In general, receptor molecules of the $\mathrm{Ig}$ and TNF superfamilies interact with ligands within the $\mathrm{Ig}$ and TNF superfamilies, respectively, but not outside of their family. HVEM 


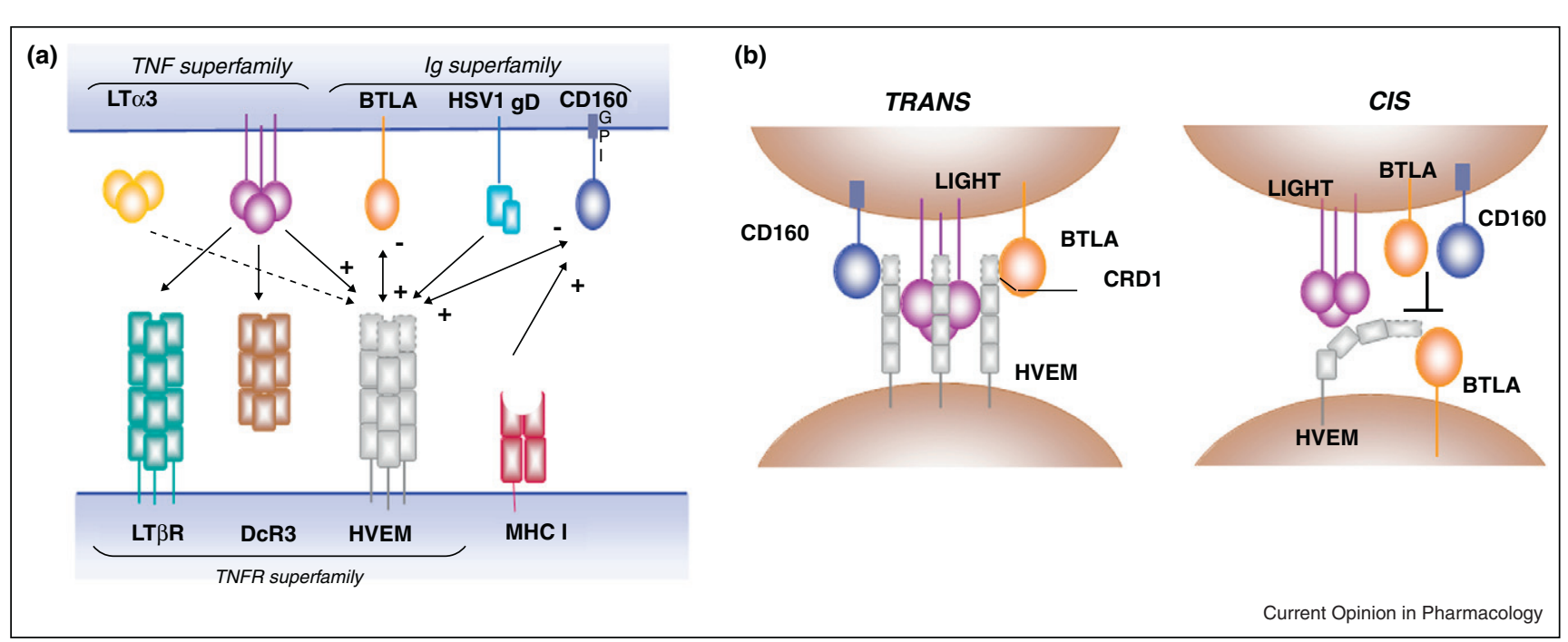

Complex binding pattern of HVEM. (a) HVEM has initially been discovered as the coreceptor for the glycoprotein D (gD) of the herpes simplex virus 1 (HSV-1), allowing the entry of the virus in the cell. The TNF-related ligands LIGHT and lymphotoxin- $\alpha 3$, and the Ig-related members BTLA and CD160, all bind to HVEM. LIGHT also binds LT $\beta$ R, another TNF receptor member, and the decoy receptor DcR3. CD160 also binds weakly to classical and non-classical MHC I molecules. The arrows indicate the specific receptor-ligand interaction. The (+) indicates co-stimulatory signaling trough ligation of LIGHT with HVEM. HVEM signaling with BTLA/CD160 is bidirectional (+/-) as BTLA and CD160 transmit inhibitory signals in cells, while BTLA and CD160 can act as activating ligands for HVEM resulting in NF-кB activation. (b) The diagram illustrates the signaling outcome for HVEM with its ligands when binding in trans (left) or cis (right) configurations. Both BTLA and CD160 bind the CDR1 domain of HVEM, whereas LIGHT interacts with a region spanning both the CRD2 and CRD3 domains. In trans configuration, binding of LIGHT to CRD2-CDR3 of HVEM does not inhibit the binding of BTLA or CD160. By contrast, the HVEM/BTLA cis complex on the same cell interferes with BTLA and CD160 binding in soluble or membrane positions for accessing and activating HVEM. Only LIGHT in the membrane can drive the dissociation of the HVEM/BTLA cis complex and is capable to activate HVEM. Soluble LIGHT enhances the binding between HVEM and BTLA but cannot activate HVEM in the cis complex.

is different, as it interacts with the viral $\mathrm{gD}$ protein and the TNF-related cytokines: LIGHT and lymphotoxin- $\alpha$ $(\mathrm{LT} \alpha)[8]$. More surprisingly HVEM was found to interact with two members of the Ig superfamily: BTLA and CD160 [1,9 $9^{\bullet}$ (Figure 1A).

\section{Binding sites on HVEM}

The extracellular domain of HVEM contains three full cysteine-rich domains (CRD), the signature motif of the TNFR superfamily [10]. The fourth C-terminal CRD has only two of the characteristic three disulfide bonds that form a CRD. BTLA binds HVEM in CRD1 at the Nterminus of HVEM $\left[11^{\bullet \bullet}, 12\right]$ in a region coined the 'DARC' side of HVEM, because it is also the attachment site for HSV gD (DARC for $\mathrm{g} \underline{\mathrm{D}}$ and BTLA binding site on the TNF $\underline{R}$ HVEM in GRD1) and CD160 [1]. CRD1 is essential for inhibitory signaling induced by HVEM as deletion of this domain abolishes the binding of BTLA and CD160, but not LIGHT, and results in costimulation by HVEM-Ig lacking in CRD1 [1]. By contrast, LIGHT and lymphotoxin- $\alpha$ bind competitively a region spanning CRD2 and CRD3 on the opposite face of HVEM $\left[12,13^{\bullet}\right]$.

The LIGHT/HVEM interaction consists of a trimeric LIGHT surrounded by an HVEM bound at the interface between each pair of LIGHT monomers thus forming a 3:3 complex typical for TNF family interactions [10]. This leaves the CRD1 of HVEM overhanging the top of the LIGHT molecule and therefore accessible for interaction with CD160 or BTLA. Binding of LIGHT to HVEM does not block either CD160 or BTLA binding. Actually, it leads to a twofold increase in CD160 or BTLA binding. Cross-blocking studies with CD160 and BTLA indicated that their binding sites on the GRD1 of HVEM overlapped to some extent. Nevertheless, CD160 and BTLA do not co-localize on the cell surface [14].

Based on the conservation seen in several TNF ligandreceptor crystal structures, the engagement of HVEM with LIGHT must be in trans (between adjacent cells). By contrast, co-expression of HVEM and BTLA on the surface of T, B and DCs cells raise the possibility of some cis interactions on the same cell. This intrinsic mechanism interferes with the ability of LIGHT in soluble form, and BTLA and CD160 in soluble or membrane form to access and activate HVEM (Figure 1B). Only membrane bound LIGHT is still capable to activate HVEM, even if activation is less important than in the absence of BTLA. Thus, BTLA functions as an inhibitor when coexpressed with HVEM. The HVEM-BTLA cis complex may provide 
intrinsic regulation in T cells serving as an interference mechanism silencing signals coming from the microenvironment [15].

\section{Dual functions of HVEM and its partners}

Interestingly, these distinct binding sites on HVEM also correlate with distinct functions. The binding of HVEM to LIGHT or LT- $\alpha$ stimulates host immunes responses, enhancing $\mathrm{T}$ cell co-stimulation, B cell costimulation in cooperation with CD40/CD40L, plasma cell differentiation and Ig secretion [16 ${ }^{\circ}$, and DC maturation [17]; while the binding to BTLA or CD160 delivers co-inhibitory signals to T $\left[6^{\bullet \bullet}, 18^{\bullet \bullet}, 19^{\bullet}, 20\right]$ and B cells [21] (and ML Thibult et al., 2012, manuscript in preparation). The discovery of inhibition via HVEM-BTLA provided an initial explanation to the paradox presented by the distinct phenotypes of the LIGHT and HVEM-deficient mice. LIGHT-deficient $T$ cells proliferated poorly in response to TCR stimulation, as expected; however, HVEM-/- T cells showed enhanced activation [22 $2^{\circ}$. Almost at the same time, BTLA was identified as the first co-inhibitory receptor of HVEM [11 ${ }^{\bullet \bullet}$ ], consistent with the hyper-responsive phenotype of HVEM-deficient T cells. Despite the complexity of ligand binding, the inhibitory function of HVEM is dominant as demonstrated by HVEM $-/-$ mice studies $\left[22^{\circ}\right]$ and the inhibitory effect of HVEM-Ig in in vitro studies [14]. CD160 functions are not clearly elucidated as some reports indicated that crosslinking of CD160 resulted in profound inhibition of human CD4+ T cells [14], whereas crosslinking of CD160 using BY55 mAb or MHC I multimers enhanced NK or CD8+ T cell cytolytic activity, as well as cytokine production (IFN- $\gamma$, TNF $\alpha$ and IL-6) $[23,24]$. Therefore, CD160 signaling certainly depends on the cell type and the extracellular domain engaged. Of note, HVEM signaling is bidirectional as BTLA and CD160 can act as activating ligands for HVEM resulting in NF$\kappa \mathrm{B}$ activation $\left[25^{\circ}\right]$.

\section{Expression patterns in normal cells}

Analysis of the HVEM network and the functional outcome of HVEM engagement with its ligands is complicated by the widespread and regulated expression of BTLA, CD160, and LIGHT (Table 1).

HVEM is widely expressed on peripheral $\mathrm{T}$ and $\mathrm{B}$ cells, and is modulated during the lymphocyte activation: HVEM is strongly present on resting T cells, downregulated upon $\mathrm{T}$ cell activation and then re-expressed as the T cells return to a more resting state. HVEM expression is high in naïve and memory B cells, but not detectable on activated $B$ cells in the germinal centers $\left[16^{\circ}\right]$. Interestingly, expression of HVEM and its ligands is reciprocal, as HVEM is decreased on DCs, B and T cells following the engagement with LIGHT $\left[16^{\bullet}, 17,26^{\bullet}\right]$ or BTLA $\left[6^{\bullet \bullet}\right]$. Besides $\mathrm{T}$ and $\mathrm{B}$ cells, HVEM is expressed on a wide range of other hematopoietic (monocytes, immature dendritic cells, Tregs, monocytes, neutrophils, and NK cells) and nonhematopoietic cells (parenchymal cells).

By contrast with the wide expression of its receptor HVEM, LIGHT expression is more restricted and tightly regulated. LIGHT is induced upon activation of CD4 and CD8 T cells, and similarly upon activation of B cells with CD40L/LIGHT signaling $\left[16^{\bullet}, 27\right]$. As LIGHT levels increase, HVEM levels coordinately decrease at approximately the same rate. LIGHT is also expressed on monocytes and is found on immature but not mature DCs. Indeed, in contrast to LIGHT induction upon $\mathrm{T}$ cell activation, LIGHT is constitutively expressed on 'immature' dendritic cells (DCs), and then down regulated shortly after maturation induced by LPS stimulation [27].

\begin{tabular}{|c|c|c|c|c|}
\hline \multicolumn{5}{|c|}{ Expression of HVEM and its ligands on normal and tumoral cells } \\
\hline \multirow[t]{2}{*}{ Molecule } & \multirow[t]{2}{*}{ Expression on normal cells } & \multicolumn{2}{|c|}{ Expression on tumors } & \multirow[t]{2}{*}{ References } \\
\hline & & Positive & Negative & \\
\hline HVEM & $\begin{array}{l}\text { T cells, B cells, NK cells, } \\
\text { Monocytes, Immature DCs }\end{array}$ & $\begin{array}{l}\text { Primary tumors: CLL, MCL, ALL, } \\
\text { Myeloma, Plasma cell leukemia, } \\
\text { Melanoma (93.75\%) } \\
\text { Cell lines: Raji Burkitt lymphoma }\end{array}$ & $\begin{array}{l}\text { Primary tumors: Melanoma (6,25\%), } \\
\text { Follicular Lymphoma ( } 26 \text { out of } 251 \\
\text { with truncations and frameshift) } \\
\text { Cell lines: DEL Hodgkin's lymphoma, } \\
\text { Daudi Burkitt lymphoma }\end{array}$ & $\begin{array}{l}{\left[4^{\bullet}, 6^{\bullet \bullet}, 31^{\bullet}, 34^{\bullet},\right.} \\
\left.35^{\bullet \bullet}, 36^{\bullet \bullet}\right]\end{array}$ \\
\hline BTLA & $\begin{array}{l}\text { T cells, B cells, DCs, } \\
\text { Myeloid cells, } \\
\text { Plasmacytoid DC }\end{array}$ & $\begin{array}{l}\text { Primary tumors: CLL, SLL } \\
\text { Microenvironment: TA-specific } \\
\text { CD8+ T cells in melanoma }\end{array}$ & $\begin{array}{l}\text { Primary tumors: MCL, Marginal zone } \\
\text { lymphoma, Follicular lymphoma, Burkitt } \\
\text { lymphoma, Hodgkin's lymphoma, } \\
\text { Melanoma }\end{array}$ & {$\left[4^{\bullet}, 6^{\bullet \bullet}, 18^{\bullet \bullet}\right]$} \\
\hline LIGHT & $\begin{array}{l}\text { Immature DCs, Monocytes, } \\
\text { Activated T and B cells }\end{array}$ & & $\begin{array}{l}\text { Primary tumors: CLL, MCL, ALL, } \\
\text { Myeloma, Plasma cell leukemia, } \\
\text { Hodgkin's Iymphoma, Melanoma }\end{array}$ & {$\left[4^{\bullet}, 6^{\bullet}, 31^{\bullet}\right]$} \\
\hline CD160 & T cells and NK cells & CLL, Hairy cell leukemia, MCL & & {$\left[5^{\circ}\right]$} \\
\hline
\end{tabular}

Abbreviations: CLL: chronic lymphocytic leukemia; MCL: mantle cell lymphoma; ALL: acute lymphoblastic leukemia; SLL: small lymphocytic leukemia; TA: tumor antigen; DC: dendritic cells; NK: natural killer. 
BTLA is expressed by lymphoid and myeloid cells, with particularly high expression by peripheral $\mathrm{B}$ cells and plasmacytoid dendritic cells (Derré et al., unpublished data) and lower expression by CD11c+ DCs and naive T cells [28 ${ }^{\circ}$. Similarly to CTLA-4, ICOS, and PD-1, BTLA is induced on $\mathrm{CD} 4 \mathrm{~T}$ cells during activation. Interestingly, BTLA is expressed on naïve CD8 T cells, and then progressively downregulated with CD8 differentiation, which is unusual for inhibitory receptors since most other inhibitory receptors are absent or low in naïve CD8 T cells and upregulated with differentiation $\left[6^{\bullet \bullet}\right]$. Moreover, BTLA remains expressed on Th1 but not Th2 cells, suggesting that BTLA may specifically downregulate Th1-mediated inflammatory responses [29].

CD160 expression is highly restricted to circulating NK cells and T cells. Within subsets of NK cells, CD160 is expressed on human CD56 ${ }^{\mathrm{dim}} \mathrm{CD} 16+\mathrm{NK}$ cells, NKT cells, and within the subsets of T cells, CD160 is found on $\gamma \delta \mathrm{T}$ cells, CD8 + CD28- T cells, a small subset of CD4+ $\mathrm{T}$ cells, and all intestinal intraepithelial $\mathrm{T}$ cells (IEL) (CD8 + CD28-CD101+). CD160 is not found on myeloid or B cells [30].

\section{Expression patterns in cancers}

Recently, some groups have reported abnormal expression of HVEM and its ligands in tumor cells or their microenvironment (Table 1).

\section{Expression in Chronic lymphocytic leukemia (CLL)}

During the past few years, our group focused on expression and function of HVEM and its ligands on normal and malignant lymphoid cells. HVEM was expressed on all normal B-lymphocytes and in most B cell malignancies [31 ${ }^{\circ}$ ]: HVEM was systematically expressed in B-chronic lymphocytic leukemia (B-CLL) and mantle cell lymphoma tested, often observed in acute lymphoblastic leukemia (ALL) and Burkitt's lymphoma (positive on Raji but absent on Daudi cell line) and absent in the Hodgkin's lymphoma cell line DEL. HVEM was not expressed in plasma cell lines, whereas it was expressed in all primary myeloma cells and in plasma cell leukemias. LIGHT was absent from all the lymphoid cells tested.

In addition to the expression of HVEM, BTLA was strongly expressed on B-CLL cells by immunohistochemistry and flow cytometry, higher as compared with other Bcell lymphomas $\left[4^{\circ}\right]$. The simultaneous expression of HVEM and BTLA in CLL cells suggests the triggering of an ineffective autocrine inhibitory loop. Moreover, we previously reported high expression of PD-1 on B-CLL cells [ $\left.32^{\circ}\right]$. The upregulation of these inhibitory receptors on CLL precursor cells could be related to the pathogenesis of B-CLL, which is considered a monoclonal expansion of antigen-selected B lymphocytes.

Interestingly, although CD160 expression is restricted to normal NK and T cells and is absent from normal B- lymphocytes, it is dramatically increased in B cell malignancies $\left[5^{\circ}, 33^{\circ}\right]$. CD160 is expressed in $98 \%$ (590/600) of CLL cases, $100 \%$ (32/32) of hairy cell leukemia, $15 \%(5 / 34)$ of mantle cell lymphoma and $16 \%$ (23/45) of other B-malignancies. In another study, all B-CLL cells also expressed CD160 (53/53) [33].

These co-signaling molecules could contribute to CLL pathogenesis. CLL cells could use the HVEM, BTLA, PD-1 inhibitory pathways to inhibit $T$ cells responses and enhance their survival. Of note, the presence of the HVEM/BTLA cis complex on the same CLL cell could prevent the trans ligation of BTLA with HVEM expressed by $\mathrm{T}$ cells, therefore the functional outcome of these coexpression is complex and the multiple trans and cis interactions have to be considered. The aberrant expression of BTLA, CD160 and HVEM in CLL makes them potential cancer biomarkers and attractive targets for immunotherapy. Moreover, this newly discovered HVEM ${ }^{\text {hi }} /$ BTLA $^{\text {hi }} /$ $\mathrm{PD} 1{ }^{\mathrm{hi}} / \mathrm{CD} 160^{\mathrm{hi}}$ phenotype may represent a new property that can be evaluated by flow cytometry to distinguish BCLL from other B cell proliferation disorders.

\section{Triggering on B cell malignancies}

On mantle cell lymphoma, engagement of HVEM increased Fas expression and enhanced the sensitivity of lymphoma cells to Fas-induced apoptosis [31 $\left.{ }^{\bullet}\right]$. This suggests that LIGHT stimulation partially reversed the resistance of lymphoma cells to Fas killing, without inducing proliferation, which could be an attractive advantage compared to the CD40/CD40L system. Since LIGHT triggering also enhances the functions of $\mathrm{T}$ lymphocytes and dendritic cells, it could be a unique way to restore an efficient tumor eradication by its pleiotropic effects on immune effectors and tumor cells.

In B-CLL cells, engagement of HVEM led to upregulation of chemokine genes such as IL-8 and IP-10, and apoptosis of leukemic cells, partly depending on the TRAIL pathway $\left[34^{\circ}\right]$. Interestingly, triggering of CD160 induced opposite effects on B-CLL cells since CD160 triggering reduced apoptosis, induced cell cycle progression and proliferation and cytokine production (IL-6, IL-8) on B-CLL cells [33].

\section{Expression in lymphoma}

By immunohistochemistry in reactive lymph nodes from benign follicular hyperplasia, BTLA was highly expressed on naïve $B$ cells of the mantle zone and to a lesser extent on memory B cells of the marginal zone $\left[4^{\bullet}\right]$. By contrast, within germinal centers B cells were uniformly BTLAnegative and follicular helper $\mathrm{T}$ cells $\left(\mathrm{T}_{\mathrm{FH}}\right)$ were BTLApositive. We have previously shown that HVEM was absent on activated B cells in the germinal center [16 $\left.{ }^{\bullet}\right]$. These findings suggest that downregulation of the HVEMBTLA pathway may play a role in germinal center B cell activation. In malignant lymphomas, we observed that 
BTLA was totally absent in follicular lymphoma ( 0 of 24 positive), mantle cell lymphoma (0 of 10 positive) and marginal zone lymphoma ( 0 of 5 positive), which was in sharp contrast with B-CLL/SLL small lymphocytic leukemia (19 of 19 positive).

\section{HVEM gene mutations in lymphoma}

Recently, Cheung and colleagues identified 46 cases $(18.3 \%)$ with non-synonymous mutations affecting TNFRSF14 (the gene coding for HVEM) in a cohort of 251 follicular lymphoma (FL) patients [35*0]. These mutations were associated with high-risk clinical features, and patients with a mutation in TNFRSF14 responded poorly to rituximab. Of interest, some of these mutations were located to amino acid residues at positions 14, 23 and 26 (corresponding to exons 1, 1 and 2, respectively), which were previously described to reduce the binding of HVEM to BTLA [13".

The effect of these mutations on HVEM/BTLA binding and function is subject of ongoing investigations. This opens the possibility of using HVEM as a prognostic marker for identifying high-risk patients, and adapt therapies accordingly. HVEM appears to be a candidate gene that might contribute to $\mathrm{FL}$ development, given the frequency of alterations, in particular mutations, in de novo FL. Another publication by Launay $e t$ al. confirmed TNFRSF14 mutations in FL, with a higher frequency ( $44 \%$ vs $18 \%$ previously), but in their cohort these alterations were not associated with poor prognosis [36 $6^{\bullet \bullet}$.

Further studies are necessary to clarify the impact of TNFRSF14 mutations on prognosis. These data, together with the finding that $\mathrm{T}_{\mathrm{FH}}$ cells are BTLA positive in FL, suggest that the extinction of HVEM by genetic alterations in lymphoma might contribute to the maintenance of $\mathrm{FL}$ microarchitecture and pathogenesis.

\section{Expression and function of BTLA and HVEM in melanoma}

Normally, BTLA is downregulated during human CD8+ T cell differentiation to effector cells. However, this is not the case for tumor-specific $\mathrm{T}$ cells as observed in melanoma patients. Despite effector differentiation, BTLA was persistently expressed, associated with limited T cell expansion and reduced IFN- $\gamma$ production. This suggests that melanoma-specific CD8 T cell responses are inhibited in vivo via this pathway $\left[6^{\bullet \bullet}\right.$. Interestingly, vaccination with peptides and $\mathrm{CpG}$ oligodeoxynucleotides bypassed this BTLA-mediated inhibition, as the tumor-antigen specific T cells downregulated BTLA.

Importantly, while HVEM was known to be expressed mainly on lymphoid cells, HVEM was found to be expressed by melanoma cells in situ and mediated functional inhibition of BTLA+ T cells. Interestingly, 50\% and $25 \%$ of metastases from patients were strongly and moderately positive for HVEM expression, respectively, whereas the remaining $25 \%$ were weakly positive or negative. Possibly, HVEM expression may correlate with disease outcome and/or define subgroups of melanoma. It was previously observed that HVEM was not expressed in plasma cell lines, whereas it was expressed in all myeloma primary cells and in plasma cell leukemias $\left[31^{\circ}\right]$. Moreover, our group showed a high expression of HVEM in a wide variety of solid tumors (unpublished data). Of note, HVEM triggering was initially described to inhibit proliferation of adenocarcinoma (HT-29) cells. All together, these data suggest that HVEM could be involved in the tumorigenesis and that outside of its costimulatory or coinhibitory role, HVEM may be considered also on the 'tumor suppressor' side.

Fourcade $e t$ al. recently showed that upregulation of BTLA and PD1 plays a role in restricting NY-ESO-1 specific CD8+ $\mathrm{T}$ cell expansion and function in melanoma $\left[18^{\bullet \bullet}\right]$. These studies further support the role of these inhibitory pathways for immune escape and in impeding effective anti-tumor $\mathrm{T}$ cell immune responses in patients with advanced melanoma. Blocking experiments showed that targeting BTLA alone or together with other coinhibitory receptors can reverse hyporesponsiveness of tumor-specific T cells from melanoma patients. Possibly, simultaneous blockade of BTLA, PD1 and CD160, may result in an even greater promotion of $\mathrm{T}$ cell activation.

\section{Therapeutic perspectives}

In the fields of autoimmunity, cell therapy and transplantation, there is great potential for the development of antibody-based and recombinant protein-based therapies to interfere with the stimulatory HVEM/LIGHT pathway and the inhibitory HVEM/BTLA/CD160 pathways. In autoimmunity and transplantation, one needs to attenuate undesirable $T$ cell responses against self-antigens and alloantigens, respectively. In the context of antitumor therapy, the goal is to enhance anti-tumor immune responses. Therapeutic blockade of the CTLA-4 or PD1/PD-L1 inhibitory pathways have shown considerable promise for the treatment of cancer patients, highlighting the potential clinical benefit of targeting co-receptors [7].

For further development, we can postulate different approaches summarized in Figure 2:

First, blocking the inhibitory BTLA pathway alone, or together with PD-1 blockage in tumor-specific lymphocytes, using antagonistic mAbs and chemical compounds. This approach could be pursued in various different cancers, as lymphoïd malignancies and solid tumors express HVEM.

Second, therapies targeting the CRD1 of HVEM to block the binding of BTLA and CD160 represent another strategy to enhance anti-tumor response. 


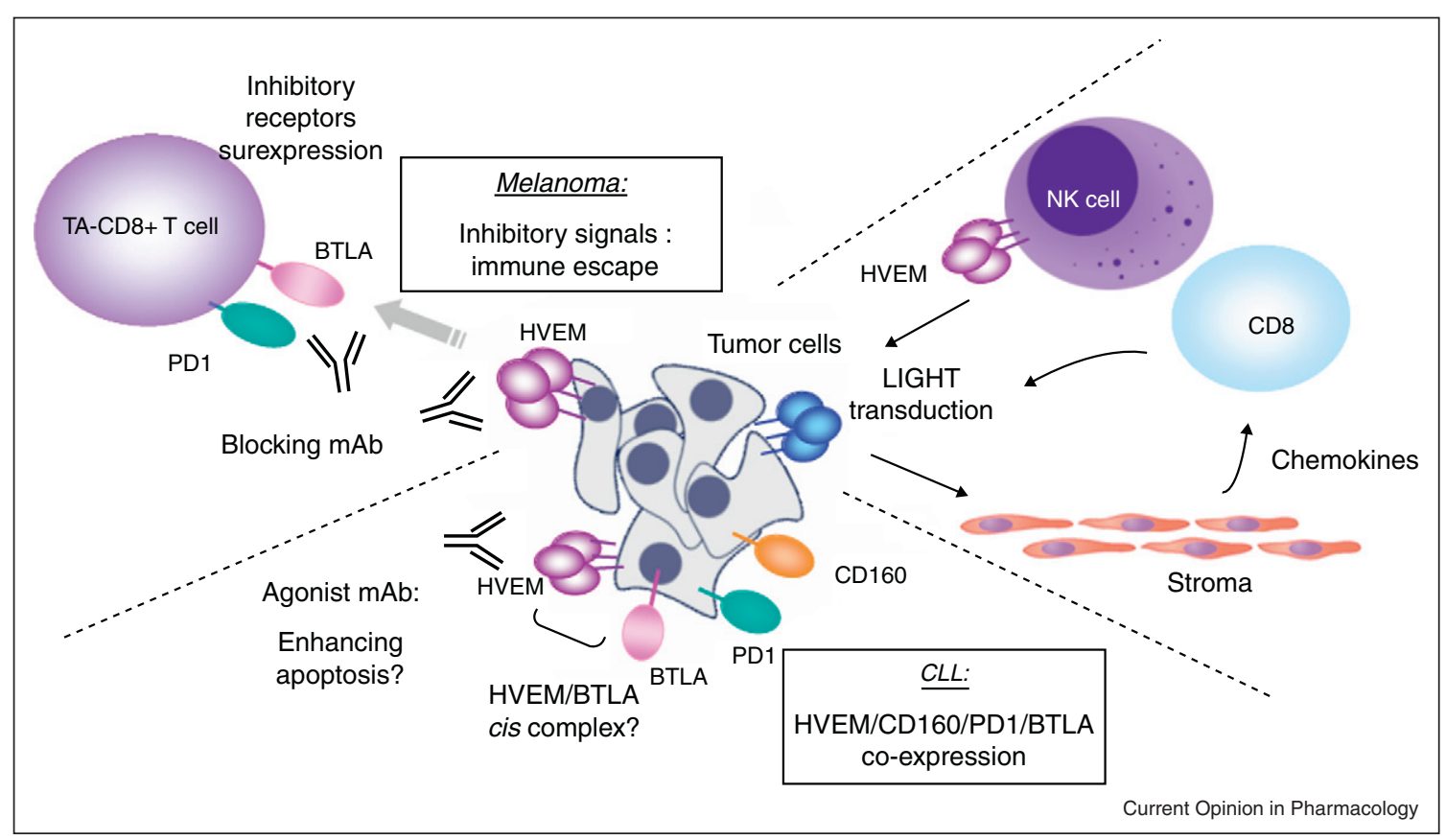

HVEM and its ligands in tumor cells: expression and possible therapeutic manipulations. We present three examples for the role of HVEM and its ligands in tumor models. In melanoma, expression and function of BTLA and PD-1 by tumor antigen-specific CD8+ T cells have been shown, probably promoting immune escape by HVEM positive tumors. Blocking mAbs against these inhibitory receptors could reverse this inhibitory function. $\checkmark$ B-CLL cells express high levels of HVEM, BTLA, PD-1 and CD160. The co-expression of these co-inhibitory receptors could contribute to CLL pathogenesis. The presence of the HVEM/BTLA cis complex on the same cell could block the trans interactions with the other ligands of HVEM expressed on T cells and the functional outcome on T cells responses has to be evaluated. Finally, expression of the costimulatory ligand LIGHT in tumors can promote anti-tumor immunity by inducing chemokine production by stromal cells, and activation of T cells and NK cells.

Third, potentiating tumor cell apoptosis, based on the finding that triggering HVEM can enhance apoptosis of mantle lymphoma cells and CLL cells mediated by death receptors.

Finally, triggering of the LIGHT/HVEM costimulatory pathway, which has been shown to act against cancer in a murine model. Specifically, LIGHT has the particularity to stimulate both stroma and T lymphocytes. LIGHT expression inside tumor cells upregulated chemokines in stroma probably through interaction with its other receptor lymphotoxin- $\beta$ receptor (LT $\beta R$ ) expressed on stromal cells, forming a microenvironment that attract naïve T cells. Then, LIGHT costimulation could prime these T cells at the tumor site, as well as NK cells, leading to eradication of tumors $\left[37^{\bullet \bullet}, 38\right]$.

\section{Conclusions}

The co-signaling molecules are crucial for regulating and maintaining efficient immune responses. Dysfunctions of the LIGHT-HVEM and BTLA-HVEM pathways are probably implied in the pathogenesis of various autoimmune and inflammatory diseases $\left[28^{\circ}\right]$. Dysregulations of this fine-tuned network are certainly also involved in neoplastic diseases.
The complexity of the HVEM network and the binding sites of the different ligands have to be considered for the development of specific antagonistic or agonistic mAbs, or recombinant soluble proteins for novel target therapies.

\section{Conflict of interest}

The authors declare no conflict of interest.

\section{References and recommended reading}

Papers of particular interest, published within the period of review, have been highlighted as:

$$
\begin{aligned}
& \text { - of special interest } \\
& \text { • of outstanding interest }
\end{aligned}
$$

1. Cai G, Freeman GJ: The CD160, BTLA, LIGHT/HVEM pathway: a bidirectional switch regulating T-cell activation. Immunol Rev 2009, 229:244-258.

2. Steinberg MW, Cheung TC, Ware CF: The signaling networks of - the herpesvirus entry mediator (TNFRSF14) in immune regulation. Immunol Rev 2011, 244:169-187.

Reviews the pro-inflammatory and anti-inflammatory roles of HVEM in experimental disease models.

3. del Rio ML, Lucas CL, Buhler L, Rayat G, Rodriguez-Barbosa Jl: - HVEM/LIGHT/BTLA/CD160 cosignaling pathways as targets for immune regulation. J Leukoc Biol 2010, 87:223-235. Highlights the role of the HVEM network in autoimmune and inflammatory diseases. 
4. M'Hidi H, Thibult ML, Chetaille B, Rey F, Bouadallah R, Nicollas R,

- Olive D, Xerri L: High expression of the inhibitory receptor BTLA in T-follicular helper cells and in B-cell small lymphocytic lymphoma/chronic lymphocytic leukemia. $A m$ J Clin Pathol 2009, 132:589-596.

Highlights the strong expression of BTLA in B-CLL cells.

5. Farren TW, Giustiniani J, Liu FT, Tsitsikas DA, Macey MG,

- $\quad$ Cavenagh JD, Oakervee HE, Taussig D, Newland AC, Calaminici M et al:: Differential and tumor-specific expression of CD160 in B-cell malignancies. Blood 2011, 118:2174-2183.

Highlights the strong expression of CD160 in B-CLL cells.

6. Derre L, Rivals JP, Jandus C, Pastor S, Rimoldi D, Romero P,

$\because \quad$ Michielin O, Olive D, Speiser DE: BTLA mediates inhibition of human tumor-specific CD8+ T cells that can be partially reversed by vaccination. J Clin Invest 2010, 120:157-167.

Identifies the upregulation of BTLA on TA-specific CD8 T cells in patients with melanoma and the unexpected expression of HVEM in melanoma cells.

7. Lesterhuis WJ, Haanen JB, Punt CJ: Cancer immunotherapy revisited. Nat Rev Drug Discov 2011, 10:591-600.

8. Ware CF: Targeting lymphocyte activation through the Iymphotoxin and LIGHT pathways. Immunol Rev 2008, 223:186201.

9. Murphy TL, Murphy KM: Slow down and survive: enigmatic

- immunoregulation by BTLA and HVEM. Annu Rev Immunol 2010, 28:389-411.

Reviews the effects of blockade of BTLA and HVEM in experimental immune models.

10. Bodmer JL, Schneider P, Tschopp J: The molecular architecture of the TNF superfamily. Trends Biochem Sci 2002 , 27:19-26.

11. Sedy JR, Gavrieli M, Potter KG, Hurchla MA, Lindsley RC

- Hildner K, Scheu S, Pfeffer K, Ware CF, Murphy TL, Murphy KM: B and $T$ lymphocyte attenuator regulates $T$ cell activation through interaction with herpesvirus entry mediator. Nat Immunol 2005, 6:90-98.

Identifies the interaction of BTLA with the TNF receptor HVEM.

12. Gonzalez LC, Loyet KM, Calemine-Fenaux J, Chauhan V, Wranik B, Ouyang W, Eaton DL: A coreceptor interaction between the CD28 and TNF receptor family members $B$ and T lymphocyte attenuator and herpesvirus entry mediator. PrOC Natl Acad Sci USA 2005, 102:1116-1121.

13. Compaan DM, Gonzalez LC, Tom I, Loyet KM, Eaton D,

- Hymowitz SG: Attenuating lymphocyte activity: the crystal structure of the BTLA-HVEM complex. J Biol Chem 2005, 280:39553-39561.

Identifies the binding sites on HVEM for its ligands.

14. Cai G, Anumanthan A, Brown JA, Greenfield EA, Zhu B, Freeman GJ: CD160 inhibits activation of human CD4+ T cells through interaction with herpesvirus entry mediator. Nat Immunol 2008, 9:176-185.

15. Ware CF, Sedy JR: TNF Superfamily Networks: bidirectional and interference pathways of the herpesvirus entry mediator (TNFSF14). Curr Opin Immunol 2011, 23:627-631.

16. Duhen T, Pasero C, Mallet F, Barbarat B, Olive D, Costello RT:

- $\quad$ LIGHT costimulates CD40 triggering and induces immunoglobulin secretion; a novel key partner in $\mathrm{T}$ celldependent B cell terminal differentiation. Eur J Immunol 2004 34:3534-3541.

Defines the role of HVEM and LIGHT in B cell physiology.

17. Morel Y, Truneh A, Sweet RW, Olive D, Costello RT: The TNF superfamily members LIGHT and CD154 (CD40 ligand) costimulate induction of dendritic cell maturation and elicit specific CTL activity. $J$ Immunol 2001 , 167:2479-2486.

18. Fourcade J, Sun Z, Pagliano O, Guillaume P, Luescher IF,

•- Sander C, Kirkwood JM, Olive D, Kuchroo V, Zarour HM: CD8+ T cells specific for tumor antigens can be rendered dysfunctional by the tumor microenvironment through upregulation of the inhibitory receptors BTLA and PD-1. Cancer Res 2012, 72:887-896.
Identifies the upregulation of BTLA on TA-specific CD8 T cells in patients with melanoma.

19. Serriari NE, Gondois-Rey F, Guillaume $Y$, Remmerswaal EB,

- Pastor S, Messal N, Truneh A, Hirsch I, van Lier RA, Olive D: B and T lymphocyte attenuator is highly expressed on CMV-specific T cells during infection and regulates their function. $J$ Immunol 2010, 185:3140-3148.

Identifies the upregulation of BTLA on CMV-specific CD8 T cells.

20. Croft M: The evolving crosstalk between co-stimulatory and co-inhibitory receptors: HVEM-BTLA. Trends Immunol 2005 26:292-294.

21. Vendel AC, Calemine-Fenaux J, Izrael-Tomasevic A, Chauhan V, Arnott D, Eaton DL: B and T lymphocyte attenuator regulates B cell receptor signaling by targeting Syk and BLNK. J Immunol 2009, 182:1509-1517.

22. Wang Y, Subudhi SK, Anders RA, Lo J, Sun Y, Blink S, Wang J,

- Liu X, Mink K, Degrandi D et al.: The role of herpesvirus entry mediator as a negative regulator of $\mathrm{T}$ cell-mediated responses. J Clin Invest 2005, 115:711-717.

First discovery of the negative role of HVEM with HVEM-/- T cells showing enhanced activation, before the identification of the interaction of HVEM with BTLA and CD160.

23. Le Bouteiller P, Barakonyi A, Giustiniani J, Lenfant F, MarieCardine A, Aguerre-Girr M, Rabot M, Hilgert I, Mami-Chouaib F Tabiasco $\mathrm{J}$ et al.: Engagement of CD160 receptor by HLA-C is a triggering mechanism used by circulating natural killer (NK) cells to mediate cytotoxicity. Proc Natl Acad Sci USA 2002, 99:16963-16968.

24. Agrawal S, Marquet J, Freeman GJ, Tawab A, Bouteiller PL, Roth P, Bolton W, Ogg G, Boumsell L, Bensussan A: Cutting edge: MHC class I triggering by a novel cell surface ligand costimulates proliferation of activated human $T$ cells. $J$ Immunol 1999, 162:1223-1226.

25. Cheung TC, Steinberg MW, Oborne LM, Macauley MG,

- $\quad$ Fukuyama S, Sanjo H, D‘Souza C, Norris PS, Pfeffer K, Murphy KM et al:: Unconventional ligand activation of herpesvirus entry mediator signals cell survival. Proc Natl Acad Sci USA 2009, 106:6244-6249.

BTLA, CD160, and herpes simplex virus envelope glycoprotein D (gD) function as activating ligands for HVEM.

26. Morel Y, Schiano de Colella JM, Harrop J, Deen KC, Holmes SD

- Wattam TA, Khandekar SS, Truneh A Sweet RW, Gastaut JA et al. Reciprocal expression of the TNF family receptor herpes virus entry mediator and its ligand LIGHT on activated T cells: LIGHT down-regulates its own receptor. J Immunol 2000, 165:43974404.

Defines the reciprocal expression of HVEM and its ligand LIGHT.

27. Pasero $C$, Truneh A, Olive D: Cosignaling molecules around LIGHT-HVEM-BTLA: from immune activation to therapeutic targeting. Curr Mol Med 2009, 9:911-927.

28. Murphy KM, Nelson CA, Sedy JR: Balancing co-stimulation and - inhibition with BTLA and HVEM. Nat Rev Immunol 2006, 6:671-681.

Reviews the co-stimulatory immune responses of engagement of HVEM with LIGHT, compared to HVEM interaction with BTLA that negatively regulate $\mathrm{T}$-cell responses.

29. Watanabe N, Gavrieli M, Sedy JR, Yang J, Fallarino F, Loftin SK, Hurchla MA, Zimmerman N, Sim J, Zang X et al:: BTLA is a lymphocyte inhibitory receptor with similarities to CTLA-4 and PD-1. Nat Immunol 2003, 4:670-679.

30. Anumanthan A, Bensussan A, Boumsell L, Christ AD, Blumberg RS, Voss SD, Patel AT, Robertson MJ, Nadler LM, Freeman GJ: Cloning of BY55, a novel Ig superfamily member expressed on NK cells, CTL, and intestinal intraepithelial lymphocytes. J Immunol 1998, 161:2780-2790.

31. Costello RT, Mallet F, Barbarat B, Schiano De Colella JM, Sainty D,

- Sweet RW, Truneh A, Olive D: Stimulation of non-Hodgkin's lymphoma via HVEM: an alternate and safe way to increase Fas-induced apoptosis and improve tumor immunogenicity. Leukemia 2003, 17:2500-2507.

Highlights the ability of HVEM to enhance the sensitivity of lymphoma cells to apoptosis. 
32. Xerri L, Chetaille B, Serriari N, Attias C, Guillaume Y, Arnoulet C,

- Olive D: Programmed death 1 is a marker of angioimmunoblastic T-cell lymphoma and B-cell small lymphocytic lymphoma/chronic lymphocytic leukemia. Hum Pathol 2008, 39:1050-1058.

Highlights the strong expression of PD1 in B-CLL cells.

33. Liu FT, Giustiniani J, Farren T, Jia L, Bensussan A, Gribben JG,

- Agrawal SG: CD160 signaling mediates PI3K-dependent survival and growth signals in chronic lymphocytic leukemia. Blood 2010, 115:3079-3088.

Defines the pro-survival role of CD160 in CLL cells.

34. Pasero C, Barbarat B, Just-Landi S, Bernard A, Aurran-

- Schleinitz T, Rey J, Eldering E, Truneh A, Costello RT, Olive D: A role for HVEM, but not lymphotoxin-beta receptor, in LIGHTinduced tumor cell death and chemokine production. Eur J Immunol 2009, 39:2502-2514.

Highlights the pro-apoptotic effect of triggering HVEM in B-CLL cells.

35.

- Cheung KJ, Johnson NA, Affleck JG, Severson T, Steidl C, BenNeriah S, Schein J, Morin RD, Moore R, Shah SP et al.:

Acquired TNFRSF14 mutations in follicular lymphoma are associated with worse prognosis. Cancer Res 2010 70:9166-9174.

Identifies high rate of acquired TNFRSF14 mutations in follicular lymphoma and their correlation with inferior clinical outcomes.

36. Launay E, Pangault C, Bertrand P, Jardin F, Lamy T, Tilly $H$,

- Tarte K, Bastard C, Fest T: High rate of TNFRSF14 gene alterations related to 1 p36 region in de novo follicular lymphoma and impact on prognosis. Leukemia 2011, 26:559-562.

Identifies high rate of acquired TNFRSF14 mutations in follicular lymphoma, not associated with poor prognosis.

37. Yu P, Lee Y, Liu W, Chin RK, Wang J, Wang Y, Schietinger A,

-. Philip M, Schreiber H, Fu YX: Priming of naive T cells inside tumors leads to eradication of established tumors. Nat Immunol 2004, 5:141-149.

Highlights the anti-tumor effect of transducing LIGHT in a murine in vivo model.

38. Fan Z, Yu P, Wang Y, Fu ML, Liu W, Sun Y, Fu YX: NK-cell activation by LIGHT triggers tumor-specific CD8+ T-cell immunity to reject established tumors. Blood 2006, 107:1342-1351. 